\title{
Spike Lee et la seconde Blaxploitation. Parabole ou naturalisme : deux stratégies testimoniales
}

Valérie Bonnet et Patrick Mpondo-Dicka

\section{(2) OpenEdition \\ Journals}

Édition électronique

URL : https://journals.openedition.org/mots/20687

DOI : $10.4000 /$ mots. 20687

ISSN : 1960-6001

Éditeur

ENS Éditions

\section{Édition imprimée}

Date de publication : 15 septembre 2012

Pagination : 29-44

ISBN : 9782847883732

ISSN : 0243-6450

\section{Référence électronique}

Valérie Bonnet et Patrick Mpondo-Dicka, « Spike Lee et la seconde Blaxploitation. Parabole ou naturalisme: deux stratégies testimoniales », Mots. Les langages du politique [En ligne], 99 | 2012, mis en ligne le 15 septembre 2014, consulté le 23 avril 2022. URL : http://journals.openedition.org/mots/ 20687 ; DOI : https://doi.org/10.4000/mots.20687

\section{(C) ENS Éditions}




\title{
Spike Lee et la seconde Blaxploitation. Parabole ou naturalisme : deux stratégies testimoniales
}

\author{
Il est clair que les œuvres de fiction en littéra- \\ ture et leurs équivalents dans les autres arts \\ jouent un rôle éminent dans la construction \\ du monde. \\ Nelson Goodman, Manières de faire des \\ mondes (2006) \\ So let's make our own movies like Spike Lee \\ Cause the roles being offered don't strike me \\ There's nothing that the Black man could use \\ to earn \\ Burn Hollywood burn. \\ Public Enemy, Burn Hollywood Burn ${ }^{1}$
}

La représentation médiatique de la communauté afro-américaine a traversé différentes étapes depuis la reconnaissance des droits civiques des Noirs au milieu des années soixante. Cette époque a connu l'avènement d'un discours d'autovalorisation, manifesté de différentes manières sur les scènes sociales, politiques (la célèbre marche vers Washington pour le travail et la liberté, menée par Martin Luther King, le 28 août 1963) et médiatiques, notamment par la musique populaire.

En ce qui concerne le cinéma, après les prémisses des films militants antiracistes, dont Sydney Poitier sera la figure de proue (avec notamment, en 1967, In the Heat of the Night de Norman Jewison et Guess Who's Coming to Dinner de Stanley Kramer), la sortie, la même année 1971, de Shaft (Gordon Parks) et surtout de Sweet Sweetback's Baadasssss Song (Melvin Van Peebles) apporte

1. Burn Hollywood Burn, extrait de l'album Fear of a Black Planet, Deflam Records, 1990.

Université Paul Sabatier Toulouse 3, LERASS

valerie.bonnet@free.fr

Université Toulouse 2 Le Mirail, LARA-CPST

pmpondo@univ-tlsez.fr 
un changement de paradigme dans la représentation cinématographique de la communauté afro-américaine, donnant lieu à ce qu'il est coutume de nommer la Blaxploitation, mouvement cinématographique inscrit dans une dynamique culturelle identitaire, imputable à la situation des Afro-Américains, qui virent les espoirs des années soixante s'estomper en même temps que le mouvement militant s'essoufflait.

Car, dans les faits, cette vague de productions cinématographique, qui déclina rapidement à partir de 1976, exploite les préoccupations raciales dans une visée commerciale, profitant ainsi des phénomènes de redéfinition des attributs, caractéristiques des groupes en situation de discrimination sociale, ainsi que l'ont montré les travaux sur les relations intergroupes.

Le terme blaxploitation a été créé au début des années soixante-dix à partir des substantifs black et exploitation par Junius Griffin, le président de la branche de la NAACP (National Association for the Advancement of Colored People) à Hollywood (Walker, Rausch, Watson, 2009), pour signifier l'exploitation des thématiques identitaires afro-américaines à travers des films montrant une image des Noirs que cette organisation juge négative. Les films relevant de cette étiquette ont pour point commun d'utiliser des acteurs et actrices noirs, de se situer dans le film de genre, voire de série B (policier, western, horreur, comédie...), de traiter du conflit entre Blancs et Noirs en attribuant aux Blancs le mauvais rôle, et, pour les films urbains, qui en sont le point de départ et la majeure partie, de montrer des personnages de prostituées, de souteneurs, en situant l'intrigue dans les milieux du banditisme.

Après une traversée du désert, entre 1976 et 1985, on assiste à une résurgence conjointe d'un cinéma noir militant/communautaire et d'un mouvement musical engagé, le hip-hop, l'un entretenant un certain nombre de connections avec l'autre - collaboration à des bandes originales, participations des artistes au casting des films (Bonnet, 2002) -, à la suite de quoi Do the Right Thing 2 (1989), de Spike Lee, constitua un facteur déclencheur ou incitatif pour un second corpus cinématographique, grâce à son succès critique et commercial. Au vu des similarités qu'il entretient avec la première période sur le plan des conditions de production, ce renouveau des films afro-américains peut être considéré comme une seconde blaxploitation ${ }^{3}$ : premier film au succès incitateur, production soutenue de films de et sur la communauté afro-américaine, sérialisation, exploitation commerciale cinématographique appuyée par une bande originale accrocheuse pour l'une, exploitation de stéréotypes communautaires et identitaires générant un succès public pour l'autre4.

2. Désormais abrégée en DTRT.

3. Désormais abrégée en $B P 2$.

4. Au nombre de ces productions, New Jack City (Mario Van Peebles, 1991), Boyz N the Hood (John Singleton, 1991), Menace // Society (Albert et Allen Hughes, 1992), Juice (Ernest R. Dickerson, 1992), Fresh (Boaz Yakin, 1994). 
Au plan de la réception, cette seconde période ne semble pas avoir suscité une réception aussi partagée que sa devancière, dont l'appréciation alla d'une forme d'expression de la black pride (Walker, Rausch, Watson, 2009, p. 136138) à un rejet épidermique.

Là où l'une, bien que campant majoritairement la délinquance des milieux urbains défavorisés, exploitait la transgénéricité5, l'autre participe de l'avènement d'un genre, le hood movie, que l'on pourrait traduire par film de ghetto ${ }^{6}$, du moins dans sa première phase. Corollaire de cette généricité, ces productions exploitent une dimension testimoniale à visée démonstrative.

A contrario, Spike Lee investit le champ du discours de la communauté noire américaine en traitant, film après film, des différents aspects internes à celle-ci, comme de ses relations à la société américaine - couple mixte (Jungle Fever, 1990), fraternité universitaire (School Daze, 1988), appel unitaire et politique à la Million March Men (Get On The Bus, 1996), réhabilitation de la figure de Malcolm X (film éponyme), rôle du sport dans l'économie sociale américaine (He Got Game, 1998), place des Noirs à la télévision (Bamboozled, 2000) - et ne reviendra au hood movie que pour s'en démarquer en jouant sur ses codes (Clockers, 1995). Les thèmes abordés montrent une communauté diversifiée, la mettent en exergue - mais également face à ses incohérences.

Si les stratégies sont différentes, il n'en demeure pas moins que des films qui traitent de la communauté afro-américaine (plus particulièrement de ses vicissitudes)7, dont les réalisateurs sont afro-américains et dont les acteurs sont conséquemment à forte majorité afro-américains, sont nécessairement reçus, quelle que soit la communauté d'accueil, comme porteurs d'un investissement qui va du message communautaire au propos politique en passant par le discours militant. Ainsi, notre problématique ne consistera pas tant à faire la démonstration de la dimension politique intrinsèque des films de Spike Lee et de ses épigones que d'analyser la façon dont la fictionnalisation de ces productions contribue à la construction de cette dimension politique.

Cependant, il est évident qu'une conscience politique sous-jacente irrigue les films de Spike Lee dans leurs moindres détails, et que la réalisation cinématographique y est conçue comme une forme d'engagement; ainsi, à propos de DTRT: «Spike était déterminé à contribuer à la chute de Koch, qui était maire de New-York depuis 1978 : 'Nous savions que le film sortirait juste avant

5. Du polar urbain (Shaft, 1971) au film de Kung Fu (Black Samurai, 1978) en passant par le film fantastique (Blackenstein, 1973).

6. Traduction qui nous paraît plus adéquate que la transposition littérale, film de quartier, dans la mesure où le quartier en question est un ghetto urbain.

7. Comme le souligne Philippe Bourgois (1997, p. 68) : «Le discours politique des années quatrevingt-dix aux États-Unis laisse peu de place à l'idéalisme des années soixante. Ainsi, l’Amérique possède un héritage historique caractérisé par une inégalité ethnique structurelle qui constitue un anathème pour sa population.» 
les primaires démocrates pour la mairie. Nous avions le sentiment d'avoir une petite influence...' ${ }^{8} 8$ (Lee, Aftab, 2005, 1976).

\section{Fictionnalisation}

\section{Du hip-hop au ghetto}

Dans le cadre de la fiction cinématographique, les films de Spike Lee ont ouvert un champ des possibles9 : l'inscription, au sein de l'hégémonie hollywoodienne, de récits sur la communauté afro-américaine pris en charge par des auteurs de cette communauté. Pour autant, ces films ne sont que des œuvres de fiction. Mais il apparaît clairement que Spike Lee joue avec la convention fictionnelle pour délivrer un discours militant sur sa communauté, stratégie qui culmine dans la biographie fictionnelle de Malcolm X.

Si les films de la $B P 2$ ont suivi la voie de la représentation interne de la communauté afro-américaine, ils ont moins subtilement joué avec les conventions fictionnelles pour constituer un genre aux modalités énonciatives et thématiques communes, le hood movie, auquel DTRT a ouvert la voie. Il convient en effet de préciser que les ghettos connaissent alors une violence endémique, 1991 étant l'année de l'apogée de l'épidémie du crack qui toucha particulièrement la population noire défavorisée. Comme le souligne Bourgois (1997, p.68), «dealers, toxicomanes et petits criminels intériorisent leur rage et leur désespoir. Ils dirigent leur brutalité contre eux-mêmes et leur communauté plutôt que vers les causes structurelles de leur oppression».

Ainsi, avant même l'avènement de la $B P 2$, la thématique existentielle déterministe (de jeunes Noirs en proie à la difficulté existentielle de leurs conditions sociales et raciales) sous-tend les films hip-hop qui apparaissent au début des années quatre-vingt (Wild Style, Charlie Ahearn, 1983; Beat Street, Stan Lathan, 1984 ; Breakin', Joel Silberg, 1984) pour accompagner l'émergence de cette culture - rap, graffiti, breakdance - et son potentiel financier, films dans lesquels la danse et/ou la musique sont l'adjuvant narratif qui permet la résolution des difficultés et servent de prétexte à une sanction positive ${ }^{10}$, un final euphorique - le plus souvent un show dans lequel les protagonistes laissent exploser leurs talents ${ }^{11}$ (topos, par ailleurs, des films musicaux).

8. "Spike was determined to contribute to the downfall of Koch, who had been mayor of New York since 1978: "We new that when the film came out it would be right before the Democratic primary for mayor. We felt that we could have a little bit of influence." "

9. Si l’on exclut Oscar Micheaux (voir Howard, 2010) ; la première Blaxploitation ne peut être considérée comme une nouvelle ouverture de ce champ des possibles, au titre qu'elle était, comme son nom l'indique, une exploitation.

10. Adjuvant et sanction au sens sémiotique de ces termes; voir Greimas, Courtés, 1993.

11. Le schéma immuable perdure dans la deuxième vague de films hip-hop, vecteurs publicitaires pour une nouvelle génération d'artistes R'n'B, tels You Got Served (Street Dancers, Sylvain 
C'est à cette culture hip-hop que Spike Lee emprunte pour mieux s'en départir, avec ironie, lorsqu'il construit le personnage de Mars Blackmon, le B-Boy de Nola Darling, stéréotype de représentation de la communauté afro-américaine que le film dynamite (voir infra) ${ }^{12}$. Dans DTRT, il n'en reste que les chaussures et les vêtements de basket; les personnages ont quitté le registre de la comédie, et même si le film se présente au premier abord comme une chronique bon enfant, le propos est ailleurs.

\section{Do the Right Thing, un modèle difficile à suivre}

$D T R T$ installe plusieurs éléments auxquels les films de la $B P 2$ emprunteront plus ou moins explicitement (à l'exception notable de la relation Noir/Blanc, que les films de la $B P 2$ évitent soigneusement) : la dimension environnementale (le quartier noir, urbain, et son emprise sur les personnages), le contexte social (précarité, fille-mère, conflit des générations), le final dysphorique, pour les traiter de manière de plus en plus conventionnelle, comme des itinéraires exemplaires et illustratifs de vérité "sociologique» générale. Les films de la PB2 se distinguent par une intentio auctoris visant à l'édification, par le truchement de la mise en scène d'une fonction testimoniale :

- les scènes d'ouverture sont sous-tendues par un fond sonore urbain émaillé de sirènes, d'échanges mimant la fréquence radio de mauvaise qualité de la police, d'interventions de reporters radio ou télé sur les lieux du délit (Boyz N the Hood, New Jack City);

- les récits s'inscrivent dans un contexte historique (émeutes de Watts en 1964 pour Menace // Society) ou social (invectives et fusillade dans Boyz N the Hood)), qui tend au déterminisme (représentation quasi-systématique du contexte social de l'enfance - Boyz N the Hood, Menace II Society, Fresh, Juice, South Central, Straight Out of Brooklyn);

- cette contextualisation peut aller jusqu'à une explicitation extradiégétique, mentions verbales incrustées à l'écran, en ouverture ou en fermeture du film. Ainsi en incipit de Boyz N the Hood: " un Noir américain survingt-et-un sera victime d'un meurtre ${ }^{13}$, ou en clôture de New Jack City, qui cumule justification véridictoire de la fiction et moralisation : «Bien que ceci soit une fiction, il existe des Nino Brown dans chaque grande ville d’Amérique. Si nous n'affrontons pas le problème de façon réaliste - sans slogans vides ni promesses - la drogue continuera de détruire notre pays. " $^{14}$

White, 2004), Stomp The Yards (Steppin', 2007), et sa suite (Steppin'2, Rob Hardy, 2010), dont, fait nouveau, les réalisateurs sont exclusivement afro-américains.

12. Parmi ces stéréotypes, le cliché raciste de la femme noire sexuellement insatiable, particulièrement cultivé par la Blaxploitation (Foxy Brown, Coffy...), que le personnage de Nola Darling, figure d'une jeune Afro-Américaine émancipée et assumant ses choix de vie, fait exploser.

13. "One out of every twenty-one Black Américain males will be murdered in their lifetime."

14. "Although this is a fictional story, there are Nino Browns in every major city in America. If we don't 
Il est intéressant de comparer ces mentions aux cartons finaux de DTRT qui, en ce qui les concerne, renvoient à deux options politiques opposées, l'autodéfense prônée par Malcolm X et la non-violence défendue par Martin Luther King. Mode d'énonciation similaire, mais intentio auctoris différentes. Alors que l'un montre, ne serait-ce que par l'apophase, le chemin à suivre, l'autre laisse au spectateur le choix de sa position. Ainsi, si Mookie, le personnage central du film, est celui par qui adviendront les troubles, la scène d'émeute est plutôt montée comme un crescendo d'exacerbation, au sein duquel chaque protagoniste répond à sa manière. Le titre du film, forme d'adresse directe au spectateur, renvoie in fine à cette alternative. Cette adresse, loin d'être fortuite, constitue un élément principal du mode énonciatif des films de Spike Lee et apparaît comme une modalité clé de sa rhétorique cinématographique.

Les films de la $B P 2$ ne reprendront jamais à leur compte ce mode et ces figures, se situant dans une forme énonciative plus conventionnelle; la voix off de Menace // Society permet tout au plus de structurer le récit rétrospectif englobant par l'entremise duquel les événements du film nous sont narrés. Les codes du récit hollywoodien y sont largement repris, à l'exception du happy ending systématisé avec l'avènement des blockbusters.

\section{Articulation politique du réel et de la fiction}

Les deux citations finales de DTRTn'ont pas une seule dimension d'interpellation et de responsabilisation; elles lient également de manière étroite le réel à la fiction. En effet, celles-ci sont mises en regard de la célèbre photographie de la poignée de main des deux leaders afro-américains cités - respectivement Malcolm X et Martin Luther King -, photographie intégrée à la diégèse par l'entremise de Smiley, le personnage mentalement déficient qui n'aura cessé d'essayer de la vendre ${ }^{15}$.

Cette fonction de liaison du réel à la fiction est également perceptible dans d'autres figures d'interpellation que sont le regard à la caméra et l'adresse verbale au spectateur (au hors-champ extra-diégétique) ${ }^{16}$, et surtout, les deux figures majeures du double dolly shot ${ }^{17}$ et du racial slur montage ${ }^{18}$. Cette sortie

confront the problem realistically - without emptyslogans and promises - then drugs will continue to destroy our country. "

15. Notons cependant que la photo que tient Smiley est marquée d'une croix rouge dans le déroulement de la diégèse, alors que la photo qui est montrée plein écran est vierge de cette croix, ce qui renforce sa dimension énonciative.

16. La figure de l'enquêteur dans Nola Darling, le wake up final de School Daze, la Love and Hate scene de Radio Raheem.

17. Il s'agit d'un travelling arrière où l'acteur est filmé sur une dolly (chariot roulant) et cadré en plan serré de façon à ce qu'il ait l'air d'avancer en flottant. L'effet est souvent utilisé au moment d'une grande introspection du personnage (par exemple, dans le film éponyme, au moment où Malcolm X se rend au Audubon Theater, dans lequel il sait qu'il a toutes les chances d'être assassiné).

18. Ainsi nommée dans le scénario de DTRT: un montage cut en plan serré où chaque personnage 
de la diégèse, rupture avec la convention cinématographique canonique, lui permet d'introduire, à divers moments de ses films, un pont entre le monde fictionnel de la diégèse et le monde réel du public; le plus souvent sur le mode de l'invective, répétée dans le cas du racial slur montage, cette adresse au spectateur, regard caméra associé à une adresse verbale, bouffée exutoire de racisme primaire, met celui-ci face à ses propres représentations raciales sans lui donner pour autant la possibilité de choisir un point de vue parmi ceux des différents protagonistes, si ce n'est celui du dernier à intervenir, qui renvoie dos à dos les précédents ${ }^{19}$. C'est notre capacité de réaction devant l'insulte qui est alors interrogée ${ }^{20}$, le regard caméra et l'invective suspendant pour un temps le contrat énonciatif qui nous fait accéder à la diégèse par l'entremise d'une instance médiatrice; notre implication dans le propos du film s'en trouve dès lors facilitée par notre insertion dans l'univers diégétique.

Enfin, c'est la diégèse qui inscrit DTRT dans un contexte contemporain : le slogan «Howard Beach » ${ }^{21}$ scandé par la foule à l'arrivée de l'escouade de policiers venus mettre fin à l'émeute succédant au meurtre de Radio Raheem, ou encore la litanie des bavures policières récentes que celle-ci, menaçante, renvoie aux policiers en train de réaliser qu'ils ont tué Radio Raheem : «Michael Stewart ${ }^{22}$, murder, Eleanor Bumpers ${ }^{23}$, murder ». Ces rappels de situations non fictionnelles, thématiquement proches et exemplaires ${ }^{24}$, soulignent qu'à l'inverse des films de la $B P 2$, ce n'est pas l'événement fictif qui a valeur d'exemple; il s'inscrit au contraire dans la filiation d'un ou plusieurs événements réels ${ }^{25}$, ce qui renforce sa valeur représentative et le naturalise.

Ainsi, chez Spike Lee, fiction et non-fiction ne cessent d'entretenir un rapport étroit, de contiguïté, ce que l'on observe de manière privilégiée à l'examen des génériques.

insulte l'un des autres groupes ethniques du melting pot américain en regard et adresse directe à la caméra, procédé repris dans 24th Hour (2002).

19. Ainsi Mister Senior Love Daddy qui, dans DTRT, appelle au calme.

20. Que nous réagissions comme cible de l'insulte, par identification (ce qui est, peut-on imaginer, le cas du public noir, asiatique ou latino) ou par empathie (dans le cas du public blanc). De ce point de vue, le film vise un public diversifié, alors que les nigga des films de la $B P_{2}$ ont une fonction plus strictement endogroupale (voir Bonnet, 2011).

21. En référence aux événements qui se sont déroulés dans ce quartier italo-américain en décembre 1986 : trois jeunes Noirs, entrés dans une pizzeria pour demander de l'aide à la suite d'un problème de voiture, sont agressés par un groupe d'adolescents pour des motifs raciaux (voir aussi Lee, Jones, 1989, p. 24).

22. Graffeur afro-américain gravement blessé au cours de son arrestation, le 15 septembre 1983, et qui mourra des suites de ses blessures.

23. Afro-Américaine de 66 ans, tuée par un policier lors de l'expulsion de son appartement, le 29 octobre 1984.

24. Peu après que le verdict de l'affaire a été rendu, le sénateur de l’État de New York, qui couvre le district d'Howard Beach, déclarait dans le New York Times : "Over the pastyear, "Howard Beach" has become the new catch-phrase for racism. "

25. Ce sont en fait les événements dont Spike Lee s'est inspiré pour écrire son scénario (voir Lee, Aftab, 2005, p. 76). 


\section{Génériques}

Spike Lee apporte un soin particulier à ses génériques : « Nous essayons toujours d'utiliser le générique d'ouverture comme un espace de concentration de l'esprit du public vers le film à venir »26 (Lee, Aftab, 2005, p. 281). Il y a dans la construction des génériques d'ouverture ou de fermeture du cinéaste un travail d'actualisation (au double sens linguistique et temporel du terme) de la problématique du film, qui aboutit à une sorte d'uchronie de l'histoire afro-américaine :

- florilège de figures et d'événements politiques de la communauté afroaméricaine (School Daze),

- montage du discours reconstruit de Malcolm X - non pas les enregistrements de Malcolm X, mais l'interprétation de Denzel Whashington - et d'extraits documentaires de la vidéo du passage à tabac de Rodney King, en montage alterné avec l'incendie progressif du drapeau américain jusqu'à l'apparition d'un X en flamme,

- diaporama macabre de photos de cadavres de jeunes Afro-Américains, minutieusement reconstituées à partir d'archives policières (Clockers),

- florilège d'images cinématographiques et télévisuelles (y compris dessins animés et catalogues d'objets, des jouets pour la plupart), qui forment par leur réunion même une collection dérisoire de représentations caricaturales des Afro-Américains (excipit et générique de fin de Bamboozled).

Chez Spike Lee, les génériques mêlant documents et reconstructions, mélangeant les temporalités, constituent un temps propre, suspendu, qui est à la fois mémoire et annonce, un temps mnésique plutôt que chronique (Zilberberg, 1988), c'est-à-dire qui se souvient des événements marquants de l'histoire afroaméricaine et prévient de leurs prolongements dans l'espace diégétique.

De ce point de vue, le film Malcolm X nous semble constituer une acmé dans l'utilisation et la maîtrise du processus de temporalisation, en jouant sur le générique d'ouverture et l'incipit, d'une part, l'excipit et le générique de fermeture, d'autre part. Alors que le générique d'ouverture invite à une lecture documentarisante (Odin, 2000) et mémorielle, l'incipit s'inscrit dans une temporalité chronique explicite (la date qui situe les événements est mentionnée à l'écran) et installe la diégèse de manière délibérément fictionnelle - un Malcolm X jeune, insouciant et haut en couleurs, dont le déplacement en compagnie de son ami Shorty (joué par Spike Lee) est manifestement chorégraphié. Le spectateur est invité, entre l'actualité documentarisante du temps mnésique du générique (Malcolm X et Rodney King relèvent d'une même actualité) et la rétrospectivité fictionnalisante de l'incipit, à intégrer la règle du jeu : ce qu'il

26. "We always try to utilize the opening-credit sequence as a space to get the audience's minds right to the film that is about to follow. » 
va voir est bien une œuvre de fiction, mais elle est en corrélation avec les préoccupations sociales contemporaines.

De même, l'excipit du film, qui commence à la fin de la séquence de l'assassinat de Malcolm X, fait alterner :

- les documents d'archives et les reconstitutions - le corps de Malcolm X emporté en urgence, la cohue devant l'hôpital où il est emmené, l'annonce du décès faite par un officier de police ;

- dans un jeu de suspension temporelle particulièrement efficace, la reprise par Ossie Davis, en voix over, de l'eulogie de Malcolm X qu'il avait prononcée lors de son enterrement;

- la séquence finale inscrivant dans une temporalité contemporaine la mémoire de Malcolm X (les enfants qui, s'adressant à la caméra, se lèvent pour affirmer chacun leur tour : "I am Malcolm X») et la corrélation avec Nelson Mandela, dont la présence ferme la boucle entre deux figures politiques de la lutte contre le racisme envers les Noirs.

Cette pluralité temporelle tend à forger une homochronie, un temps unifié qui réunit les figures de la lutte antiraciale et permet au biopic de dépasser le cadre de l'évocation biographique pour l'inscrire dans l'Histoire, et au-delà, en construire la mythologie.

Au regard de cet usage maîtrisé et complexe de l'énonciation temporelle cinématographique, les films de la $B P 2$ donnent le sentiment d'une grande conformité. À titre d'exemple, l'ensemble générique/incipit qui nous paraît le plus élaboré (Menace // Society) est constitué d'un prégénérique in medias res qui s'achève sur une voix off, suivi d'une séquence à fonction véridictoire qui montre les scènes d'émeutes du ghetto de Watts comme cadre sociospatial de l'univers diégéLà où, chez Spike Lee, chaque générique tente de construire une temporalité synthétique qui soutient le discours d'actualisation de la problématique politique (raciale, communautaire, militante) du film, les films de la $B P 2$ assemblent au mieux une temporalité composite juxtaposant, sans les fusionner, les différents éléments avec lesquels ils exposent leur propos.

\section{Auteur, acteurs et personnages}

Ce rapport complexe entre monde réel et univers fictionnel est central dans la construction des personnages de Spike Lee. Par-delà le rôle attribué à Ossie Davis, acteur et figure de la lutte de la communauté afro-américaine, voix over (re) lisant l'eulogie qu'il avait écrite et prononcée à l'enterrement de Malcolm X dans le biopic consacré au leader noir (voir supra), on peut percevoir dans la synthèse que constituent les protagonistes de ses films une galerie de portraits hauts en couleur. 
Ceux-ci associent de manière singulière des traits de la culture cinématographique ${ }^{27}$, de la culture populaire, voire du fonds mythique ${ }^{28}$. On retrouve dans cette collection des figures qui oscillent entre mythes littéraires et archétypes, et dont la caractéristique commune est de dépasser le seul horizon d'attente du film de ghetto29 pour intégrer la tragédie grecque ${ }^{30}$, le cinéma hollywoodien ${ }^{31}$, le panthéon communautaire. Ces personnages que nous qualifierons d'archétypaux 32 constituent des figures, au sens sémiotique du terme (Courtés, Greimas, 1993, p. 148), qui fonctionnent comme des contre-stéréotypes, en ce que la dimension stéréotypique de certaines de leurs caractéristiques saillantes (Radio Raheem, identifié à son ghetto blaster, Left Hand Lacey, le pianiste de Mo'Better Blues, toujours en retard) est invalidée par d'autres de leurs attributs (l'un est un B-Boy qui reproduit les scènes de prêche, l'autre est métis et vit avec une non-Afro-Américaine).

En revanche, les personnages de la $B P 2$ constituent une forme de galerie de stéréotypes qui semble être le corollaire de la dimension générique de ces productions : mère-courage célibataire, meilleur ami de mauvais conseil, père souvent absent, grands parents religieux et respectueux de la morale.

Si les deux principes de composition des personnages divergent, c'est en terme de traitement de la réalité : le stéréotype est une épure, les psychologues sociaux parlent à son propos d'avarice cognitive, et c'est en ceci qu'il est un puissant indice de lisibilité du discours33, car il contribue à la constitution d'un code sémiotique; à l'inverse, l'archétype est une condensation. Comme le stéréotype, l'archétype est doté de signifiance, à ceci près que celle-ci n'est pas de l'ordre de la codification, mais du questionnement 34 .

De par leur dimension psycho-sociale et langagière (ici au sens de langage

27. Sal, l'Italo-Américain amateur de base-ball et propriétaire d'une pizzeria, qui ne peut qu'évoquer les films de mafia newyorkaise; Radio Raheem, le B-Boy qui porte des bagues Love/Hate et renvoie au révérend Harry Powell de La nuit du chasseur.

28. Da Mayor, l'alcoolique qui recommande de faire toujours ce qui est juste (the right thing) ou Smiley, le déficient mental qui survit grâce à la vente de photos de Martin Luther King et Malcolm $X$ constituent les deux pendants de la figure mythique de la folie associée à la sagesse : le fou et l'innocent.

29. Cette figure du fou porteur de sagesse est également présente dans le cinéma russe.

30. Mister Senior Love Daddy, l'animateur radio, constitue le chœur de DTRT, fonction qui peut également être attribuée à Smiley puisque les photos que vend celui-ci renvoient à la question finale de l'emploi de la violence ou du pacifisme.

31. Ainsi Nola Darling, construit sur la forme d'une enquête à la manière de Citizen Kane.

32. Parmi les nombreuses définitions de cette notion assez polysémique, nous reprendrons celle de Jung (La psychologie de l'inconscient). Pour une définition précise et concise, voir Lalande, 1991, p.1236.

33. «En tant que représentation collective accréditée, le stéréotype relève du fonds commun à partir duquel un groupe donné façonne sa vision des choses et des événements. Il offre les schèmes grâce auxquels la communauté peut appréhender le réel de façon uniforme et fonder en vérité ses croyances. » (Amossy, 1991, p. 48).

34. Selon Jung, les archétypes sont des types originels de représentations symboliques, servant des modèles de comportement, que renferme l'inconscient. 
cinématographique), les stéréotypes possèdent une forte dimension sociale (Leyens, Yzerbyt, Schadron, 1996, p. 24), alors que les archétypes sont avant tout un lieu de la connexion entre l'individuel et le collectif, confinant à l'universel 35 ; les uns sont une simplification de la réalité, les autres une conjonction des strates sédimentaires de l'imaginaire. Si l'un confine à l'interchangeabilité, la reproduction à l'identique de film en film (sens premier de stéréotype), l'autre renvoie à une intertextualité36. Pour reprendre la dichotomie de P. Ricœur, s'oppose ici une identité idem à une identité ipse.

Le second point de divergence est la différence de construction du personnage central : si, dans la $B P 2$, le héros répond aux canons du genre et n'échappe pas à la stéréotypie qui touche les personnages secondaires (jeune homme dont le destin est forgé par le déterminisme social et dont la chute est précipitée par l'entourage), chez Spike Lee, le héros suit les contours d'une construction psychologique plus fouillée et moins frappée par l'esthétique créative. Cette figure centrale, autour de laquelle s'organise l'univers fictionnel (Mookie dans DTRT, Nola et Malcolm X dans le film éponyme, Delacroix dans Bamboozled), ne sont pas des personnages manichéens, mais des Janus qui juxtaposent bons et mauvais choix (Mookie est à l'origine de l'émeute qui provoquera la destruction de la pizzeria de Sal, qui est pourtant son ami, comportement qui le laissera plein d'amertume à la fin du film). La différence entre le héros et son entourage, à la caractérologie plus rudimentaire 37 , tend à défictionnaliser le personnage central38.

À une collection de personnages hétérogènes au mode de construction variable répond donc un groupe cohérent, qui se caractérise par ce que la psychologie sociale nomme l'entitativité, c'est-à-dire la capacité à former une entité.

Pour Campbell (1958), à l'origine de cette notion (voir Morchain, 2005), trois critères président à cette entitativité : le partage d'un destin commun, la ressemblance et la proximité physique. Ainsi, les personnages de la $B P 2$, circonscrits au contexte géographique des ghettos noirs emblématiques (Watts, South Central, Harlem), ont un destin interchangeable (délinquance, mort violente, etc.). L'entreprise de dénonciation de la $B P 2$ participe donc au renforcement de stéréotypes sociaux. Le modèle identitaire qu'elle propose, fortement empreint de déterminisme social, possède deux fonctionnalités : il permet de fonder ou renforcer l'identité de groupe (Amossy, 1991, p. 36) tout en offrant une forme de

35. A contrario, l'archétype appartient à l'inconscient.

36. Comme le souligne Lalande (1991), la notion d'archétype renvoie essentiellement à l'idée de modèle opposé à ses copies. Le personnage de Smiley et son avatar (à la signifiance mythique près), le Duh Duh Man de New Jack City sont à ce titre exemplaires.

37. Ceux-ci constituent une forme d'exemplarité en ce sens qu'ils sont représentatifs d'une catégorie. En effet, les films de Spike Lee ont une visée démonstrative et supposent, au regard de cette visée, que soit effectuée une forme de réductionnisme.

38. Ce procédé est aussi perceptible dans le choix des acteurs, qui va souvent à l'encontre d'un casting évident. Ainsi une figure montante de Hollywood, dont la filmographie tend à lui construire une carrière de jeune premier, Denzel Washington, a-t-il été choisi pour incarner Malcolm X, personnalité controversée de la militance afro-américaine. 
stabilité rassurante. Comme l'ont montré les travaux de Tajfel et Turner en psychologie sociale, la perception des relations statutaires entre le groupe dominant et le groupe dominé génère une identité sociale sécure si celles-ci sont perçues comme immuables, et insécure si ces relations sont remises en question.

À l'inverse, Spike Lee déstabilise le rapport individuel à l'endogroupe par la logique de questionnement qui est la sienne (emblématiquement, le final de $D T R T$ ), interrogeant ainsi l'identité sociale. Sa marque pointe néanmoins dans les productions de ses successeurs : le schéma actanciel des films de la BP2 souligne la responsabilité de la communauté sur son propre destin (car c'est toujours un Afro-Américain qui provoque la déchéance du héros). Cependant est omise la notion de choix, caractéristique de l'organisation discursive du réalisateur de Mo'Better Blues, pour lui substituer un fatalisme à valence victimaire.

En bref, alors que la $B P 2$ se veut dénonciatrice et construit, pour chaque film, un exemple des faits qu'elle entend dénoncer, ce en quoi elle rejoint certaines orientations de sa devancière (Shaft in Africa), Spike Lee, lui, renvoie la réflexion au spectateur : "Do the right thing », comme l'indique le titre de l'opus; mais l'appréciation du référent du lexème right revient à ce dernier, qui se voit enjoindre de choisir. En effet, si le destin des personnages centraux, mais surtout leur ambivalence, bloque le mécanisme identificatoire - l'un parce que le public de Spike Lee n'est pas un public communautaire, l'autre parce que les personnages ne sont justement pas des stéréotypes, dont l'une des fonctions est l'adhésion et le renforcement de l'identité sociale39 (voir note 14) -, cette dernière n'en suscite pas moins une forme d'empathie. In fine, le discours politique du réalisateurvise à montrer que le Noir est un individu par-delà toute appartenance communautaire, cette démonstration passant par sa non-idéalisation, le dévoilement des faiblesses et une mise en questionnement du spectateur. En plaçant ce dernier face au choix du héros et en le renvoyant à ses propres réponses, c'est-à-dire à sa propre responsabilité, Spike Lee surmonte le travers d'un discours adressé à sa seule communauté et interroge un auditoire qu'il veut composite40.

Bien loin de proposer des stratégies individuelles, il propose ainsi un dépassement des stratégies communautaires 41 empreintes de déterminisme qu'ont choisies ses suiveurs. Le discours de ses films, qui illustrent une trajectoire personnelle ou individuelle humanisée, vient alors compléter ses interventions publiques pro-afro-américaines, qui visent à faire entendre que le Noir américain fait partie intégrante du paysage culturel dans tous ses aspects, et non seulement en tant que communauté stigmatisée.

39. En effet, le stéréotype, qu'Amossy envisage comme une construction de lecture, dépend d'un calcul interprétatif et de connaissances encyclopédiques et n'apparaît « qu'à l'observateur critique ou à l'usager qui reconnaît spontanément les modèles de sa collectivité » (Amossy, 1991, p. 21).

40. Voir à ce propos Lee, Wiley, 1992, p. 4 .

41. Il refuse également l'essentialisation qui fut une des stratégies communautaires d'affirmation d'une fierté noire (voir Bonnet, 2011). 


\section{Blaxploitation 2, deuxième période}

Après la première vague des films sortis à la suite du succès de DTRT, et qui constitue notre corpus de travail, le crédit (au double sens artistique et financier) des réalisateurs de ces films s'amenuise au gré de leurs réalisations, les conduisant à adopter diverses tactiques de maintien au sein de la production hollywoodienne. Voit alors le jour un second ensemble de films qui assoit notre hypothèse de seconde blaxploitation, dans la mesure où l'on y retrouve, accrues, les atténuations du discours politique, identitaire et/ou communautaire des films pionniers au bénéfice des stéréotypes raciaux, le recours aux facilités narratives et discursives du film de genre, la transgénéricité, le tout au détriment de la qualité discursive des œuvres. Cette modification du propos va de pair avec une augmentation significative des budgets alloués aux films. Mais la principale différence avec la première Blaxploitation demeure, dans un premier temps : ce sont des réalisateurs afro-américains qui sont à la tête de ces films.

La $B P 2$ avait connu un démarrage fulgurant lors de l'année 1991, qui vit l'émergence d'un nombre incroyable 42 de films réalisés par des AfroAméricains : avec Boyz'N the Hood (John Singleton), New Jack City (Mario Van Peebles), Straight out of Brooklyn (Matty Rich), Hangin' with the Home Boys (Joseph B. Vazquez), ce sont «pas moins de dix-sept films réalisés par des Africains-Américains [qui] sortent sur les écrans du pays, constituant ainsi un record en la matière» (Dubois, 2001, p. 37).

Pour leur seconde réalisation, le discours des premiers auteurs de la $B P 2$ connaît un progressif recul militant, dans le même temps que les films s'ouvrent à une diversité de genres : la comédie romantique pour John Singleton (Poetic Justice, 1993) et Matty Rich (The Inkwell, 1994), le western pour Mario Van Peebles (Posse [La revanche de Jesse Lee], 1993). De nouveaux venus apparaissent alors, reprenant les recettes récemment éprouvées par leurs prédécesseurs (Juice, d'Ernest Dickerson, 1992, Menace II Society, des frères Hugues, 1994), ou usant des ficelles du genre (Rage in Harlem, 1991, et Deep Cover, 1992, de Bill Duke), tandis que déjà les ingrédients du hood film sont repris par les studios et réalisés par des Blancs, dans la plus pure tradition de la première blaxploitation (South Central de Steve Anderson, 1992, produit par Oliver Stone pour Warner Bros, ou Fresh, de Boaz Yakin, 1994). Mais les réalisateurs nouvellement arrivés ont en tête l'exemple de Spike Lee, qui sort Malcolm $X$, son sixième film, à la même période (1992). Ainsi, plus pragmatiques que leurs aînés du cinéma noir indépendant des années quatre-vingt (tels qu'Haïlé Gerima, Charles Burnett, Bill Woodberry, Larry Clark...) qui tentèrent, sans grand succès, de développer une production et une distribution totalement indépendante d'Hollywood (Dubois,

42. Au regard de la production exsangue de films afro-américains lors de la décennie précédente. 
2001, p. 33), les cinéastes de la $B P 2$ vont essayer, tour à tour, de sortir de l'ornière du hood movie en visant d'autres genres et de réduire leurs prétentions discursives et identitaires, pour rester à Hollywood malgré les possibles échecs, forme de constante hollywoodienne qui fonctionne à l'intégration sur le court terme, à l'assimilation sur le long terme43.

Ainsi, les tenants du discours de revendication identitaire se raréfient. Matty Rich, annoncé comme la relève de Spike Lee, quitte Hollywood après son second film; Mario Van Peebles reprend sa carrière d'acteur, de réalisateur pour la télévision, et ne doit de rester en relation avec le cinéma et les thématiques identitaires qu'à la capitalisation de l'héritage paternel (Panther, 1995, sur un scénario de Melvin Van Peebles; How to Get the Man's Foot Outta Your Ass, sur la réalisation de Sweet Sweetback Badasssss song, où il joue le rôle de son père). Cependant, la situation n'est plus la même : bien qu'en petit nombre, les réalisateurs afro-américains ont réussi à infiltrer Hollywood et, au prix de quelques ajustements stratégiques, à s'y maintenir. Le mouvement est aussi externe au microcosme des premiers réalisateurs de hood movies; apparaissent alors de nouveaux films, aux propos moins revendicatifs (notamment de nombreuses comédies romantiques) et mettant en scène la communauté afroaméricaine ; les acteurs afro-américains passent derrière la caméra pour développer des projets tout aussi tournés vers la communauté (Waiting to Exhale [Où sont les hommes], de Forest Whitaker, Antwone Fischer, 2002 et The Great Debatters, 2007, de Denzel Washington). La cause de la représentation des Noirs américains par des membres de leur communauté est semble-t-il entendue. Reste que la teneur du discours demeure sujette à discussion - témoin le dernier dialogue tendu, par médias interposés, entre Spike Lee et Tyler Perry, le réalisateur afro-américain le plus bankable du moment, dont Spike Lee qualifie les films, il est vrai lénifiants, de coonery, buffoonery (Bogle, 1994)

Si l'ensemble du travail de Spike Lee s'inscrit dans son discours politique adossé à une relation ambivalente entre fiction et réalité, ce vraisemblable persuasif qu'Aristote évoquait dans sa Poétique, les deux films les plus importants quant à l'avènement d'une seconde Blaxploitation sont DTRT et Malcolm X, le premier comme inaugural, le second comme acmé. De la fiction librement inspirée d'un fait réel (DTRT) au biopic (Malcolm X), Spike Lee montre la voie d'un discours sur la communauté affranchi des stéréotypes endogroupaux ou des poncifs communautaires - voie que n'auront pas suivie les films de la $B P 2$. Cela valide selon nous l'usage, les concernant, du terme de Blaxploitation, celle-ci étant plus marquée encore lorsque l'on évoque les films suivants des réalisateurs qui ont émergé après la sortie de DTRT.

La position auctoriste de Spike Lee et son utilisation de la fictionnalisa-

43. Voir les trajectoires de Lee Tamahori, Mira Naïr, voire de Roberto Rodriguez. 
tion, qui oscille entre vérité fictive (fictionnelle) et vérité du monde réel (Baroni, 2009), s'oppose à la doxa que la $B P 2$ contribue à construire par sa généricité, mais également à laquelle elle fait appel par divers artefacts à caractère testimonial.

De la même manière que la première Blaxploitation suivait le chemin emprunté par le Sweet Sweetback's Baadasss's Song de Melvin Van Peeble sans jamais en retrouver la subversion esthétique et politique44, la seconde Blaxploitation suit le sillage de Do the Right Thing, sans en atteindre la portée de fiction politique; de fait, les réalisateurs afro-américains de la seconde Blaxploitation auront du mal à assumer la filiation du film de Spike Lee, tant dans leurs filmographies que dans leurs discours, à l'exception notable de John Singleton, qui répète au fil des interviews l'importance de Spike Lee pour l'ensemble des Afro-Américains qui travaillent à Hollywood:

Beaucoup de gens doivent se rendre compte que ce métier ne serait pas le même sans la carrière et les efforts de Spike. [...] Maintenant, beaucoup de gens veulent l'avoir facile, vous voyez ce que je veux dire, ils ne comprennent pas tout ce vieux truc d'avoir de la reconnaissance pourvraiment y arriver dans ce métier. Vous devez vraiment être reconnaissant, vous devez avoir de la volonté, vous devez le sentir jusqu'au sang, à la sueur, aux larmes. 45

\section{Références}

Amossy Ruth, 1991, Les idées reçues. Sémiologie du stéréotype, Paris, Nathan.

ArIstote, 1997, Poétique, Paris, Les Belles Lettres.

Autin Frédérique, 2009, "La théorie de l'identité sociale de Tajfel et Turner», en ligne [http://www.prejuges-stereotypes.net/main.htm], consulté le 2 juillet 2011.

BARONI Raphäel, 2009, "Regarder le monde en face?», en ligne [http://www.voxpoetica.org/t/articles/baroni.html], consulté le 2 juillet 2011.

Bogle Donald, 1994, Toms, Coons, Mulattoes, Mammys and Bucks, an Interpretive History of Blacks in American Films, Oxford, Roundhouse.

BONNET Valérie, 2002, "Revendication et politiques en paroles. Chansons de la communauté noire américaine», Mots. Les langages du politique, nº 70, p. 65-78.

- 2011, "Don't call me nigger, Whitey. Autodésignation de la communauté afroaméricaine et construction identitaire », Communication, $\mathrm{n}^{0} 28$ (2), en ligne [http:// communication.revues.org/index1803.html], consulté le 10 janvier 2012.

44. "Ce qu'a fait Hollywood, c'est supprimer le message politique et ajouter la caricature », explique Van Peebles, «et la Blaxploitation est née» (voir Lambert Craig, «The Blaxploitation era», The Harvard Gazette, janvier-février 2003).

45. "A lot of people need to realise that this business would not be the same if it wasn't the career and efforts of Spike. [...] A lot of people now want it really easy, you know what I mean, they don't understand the whole old school thing of paying your dues to really make it in this bussiness. You really have to pay your dues, you have to want it, you have to feel it blood, sweat, and tears. " Voir [http://www.youtube.com/watch?v=obVWjd89XhA], notre transcription. 
BouRgoIs Philippe, 1997, "Résistance et autodestruction dans l'apartheid américain ", Actes de la recherche en sciences sociales, nº 120, p. 60-68.

BRUNEL Pierre éd., 1988, Dictionnaire des mythes littéraires, Paris, Rocher.

CAMPBELL Donald T., 1958, "Common fate, similarity and other indices of the status of aggregates of persons as social entities », Behavioural sciences, vol. III, p.14-25.

CourtÉs Joseph, Greimas Algirdas-Julien, 1993 [1976], Sémiotique. Dictionnaire raisonné de la théorie du langage, Hachette ( $2^{\mathrm{e}}$ édition).

Duвoıs Régis, 2001, Dictionnaire du cinéma afro-américain. Acteurs, réalisateurs, techniciens, Paris, Séguier.

Guerrero Ed, 1993, Framing Blackness, Philadelphia, Temple University Press.

Goodman Nelson, 2006, Manières de faire des mondes, Paris, Gallimard (Folio/Essais).

Howard Ron J., 2010, Faces in the Mirror. Oscar Micheaux and Spike Lee, Lady Lake, Fireside.

LALANDE André, 1991, Vocabulaire technique et critique de la philosophie, Paris, PUF.

LEE Spike, WILEY Ralph, 1992, By any Means Necessary. The Trials and Tribulations of the Making of Malcolm X, New York, Hyperion.

LEE Spike, AfTAB Kareem, 2005, That's my Story and I'm Sticking to it, Londres, Faber and Faber.

LeE Spike, Jones Lisa, 1989, Do the Right Thing, a Spike Lee Joint, Lady Lake, Fireside.

LeYENS Jacques-Philippe, Yzerbyt Vincent, Schadron Georges, 1996, Stéréotypes et cognition sociale, Liège, Mardaga.

MoRChAIn Pascal, 2005, "Stéréotypes, stéréotypisation et valeurs», en ligne [http:// www.prejuges-stereotypes.net/main.htm], consulté le 2 juillet 2011.

Odin Roger, 2000, De la fiction, Bruxelles, De Boeck Université.

Ricoeur Paul, 1990, Soi-même comme un autre, Paris, Le Seuil.

WEINSTEIN Jeremy S., 1988, "The media have wronged Howard Beach», New York Times, 30 janvier 1988 .

RHINes Jesse A., 1996, Black Film, White Money, Chapel Hill, Rutgers University Press.

Walker David, Rausch Andrew J., Watson Chris (interviewers), 2009, Reflections on Blaxploitation. Actors and Directors Speak, Lanham, Scarecrow Press.

ZILBERBERG Claude, 1988, «Le rythme revisité», Cahiers de sémiotique textuelle, nº 14 , p. 25-36. 